\title{
Polarization and Color Filtering Applied to Enhance Photogrammetric Measurements of Reflective Surfaces
}

\author{
Jeffrey M. Wells ${ }^{1}$, Thomas W. Jones ${ }^{2}$, Paul M. Danehy ${ }^{3}$ \\ Advanced Sensing and Optical Measurement Branch \\ NASA Langley Research Center, Hampton, VA 23681
}

Techniques for enhancing photogrammetric measurement of reflective surfaces by reducing noise were developed utilizing principles of light polarization. Signal selectivity with polarized light was also compared to signal selectivity using chromatic filters. Combining principles of linear cross polarization and color selectivity enhanced signal-to-noise ratios by as much as 800 fold. More typical improvements with combining polarization and color selectivity were about 100 fold. We review polarization-based techniques and present experimental results comparing the performance of traditional retroreflective targeting materials, cornercube targets returning depolarized light, and color selectivity.

\section{Nomenclature}

$\begin{array}{lll}B R & = & \text { both cameras, LEDs on the right side only } \\ B L & = & \text { both cameras, LEDs on the left only } \\ L L & = & \text { left camera only, LEDs on left side } \\ R R & = & \text { right camera only, LEDs on right side } \\ \text { color } & = & \text { color selectivity } \\ \text { sphere } & = & \text { microsphere retroreflectors with coaxial aligned polarization } \\ C C & = & \text { microcornercube retroreflector with coaxial cross polarization } \\ U & = & \text { unpolarized } \\ P & = & \text { polarized } \\ L E D & = & \text { light emitting diode } \\ \mathrm{P}_{\mathrm{V}} & = & \text { vertically polarized } \\ \mathrm{P}_{\mathrm{H}} & = & \text { horizontally polarized }\end{array}$

\section{Introduction}

A new generation of ultra-lightweight and inflatable "gossamer" space structures, solar sails, and solar collectors are being developed to minimize space cargo packing size and weight. Noncontact methods are preferred for operational testing of ultralightweight structures. Optical shape characterization of gossamer structures has previously been reported using the method of photogrammetry. However, photogrammetry of both aluminized and transparent gossamer structures are often hampered by the reflection of

\footnotetext{
${ }^{1}$ National Research Council Associate, mail stop 238, ${ }^{2}$ mail stop 238, ${ }^{3}$ Associate Fellow AIAA, mail stop 493 Job Title, Department Name, Address/Mail Stop, and AIAA Member Grade ${ }^{1}$
} 
illumination light directly into the recording cameras. This paper aims to overcome this limitation of photogrammetry.

Photogrammetry is an optical measurement technique for deriving 3D surface data from recorded still frame or video images. Triangulating intersecting rays acquired from multiple perspectives to a point on the test article provides corresponding $\mathrm{x}, \mathrm{y}$, and $\mathrm{z}$ coordinates for that point. Under good circumstances, photogrammetry may return a measurement accuracy of one part in 70,000 of the test article's dimensions. A key requirement in photogrammetric measurement is high-contrast targets, allowing sub-pixel centroid determination by photogrammetry software. Attached retroreflective targets provide a high return of light back in the same direction of incidence. These highcontrast targeting materials are used extensively in close-range photogrammetric applications, and are considered the optimal standard for photogrammetric targeting.

A substantial hindrance to accurate centroiding of targets applied on shiny surfaces such as proposed solar sails, solar collectors or space antennae is the presence of unwanted glints of specular reflection from shiny surfaces back towards the camera. Unwanted glints can cause saturation of camera pixels, which obscures targets. Standard photogrammetric software often cannot re-identify individual targets again after they temporarily become obscured by glints. This results in fewer measured data points on the test article surface. The presence of glints is common in photogrammetric measurements of reflective surfaces, thus creating a need for glint removal. To improve signal-to-noise ratios for retroreflective targets on reflective gossamer membranes, we tested traditional retroreflective targeting materials, cornercube targets returning depolarized light, and color selectivity with monochromatic LED arrays in conjunction with chromatic filters.

Traditional retroreflective tape consisting of thousands of transparent microscopic spheres is the most common targeting material used in close-range photogrammetric applications. Light incident upon the surface is internally reflected from the back surface of the microsphere and returns outward through the front surface. More recently, a new type of retroreflective tape possessing a micro cornercube layer has become commercially available $\left(3 \mathrm{M}^{\mathrm{TM}}\right)$. These retroreflectors have minute prisms that reflect light back in the direction of the light source. Due to the geometry and optical imperfections of these microprisms, light reflected back from the cornercubes has a random polarization orientation, even when illuminated by linearly polarized light ${ }^{2}$. This effect is exploited for the coaxial crossed polarization noise reduction scheme described below.

\section{Principles of polarization noise reduction}

Linear and circular polarizing filters are commonly used in outdoor photography to reduce sunlight glare. Linear polarization filters selectively transmit one axis of polarization, while either reflecting or absorbing the perpendicular axis. If a second linear polarizer is placed in series, the maximum transmission of light occurs when the polarization axes of the two polarizers are parallel. Minimal transmission occurs when their axes are perpendicular. This eliminates nearly all specularly reflected light from the recorded images. Figure 1 depicts the polarizer setup and the reflective properties of microsphere, specular (mirror-like), and cornercube surfaces when illuminated with linearly polarized light. Microspheres and mirrored surfaces return the same polarization orientation, while the micro cornercube reflectors randomly depolarize the orientation. 


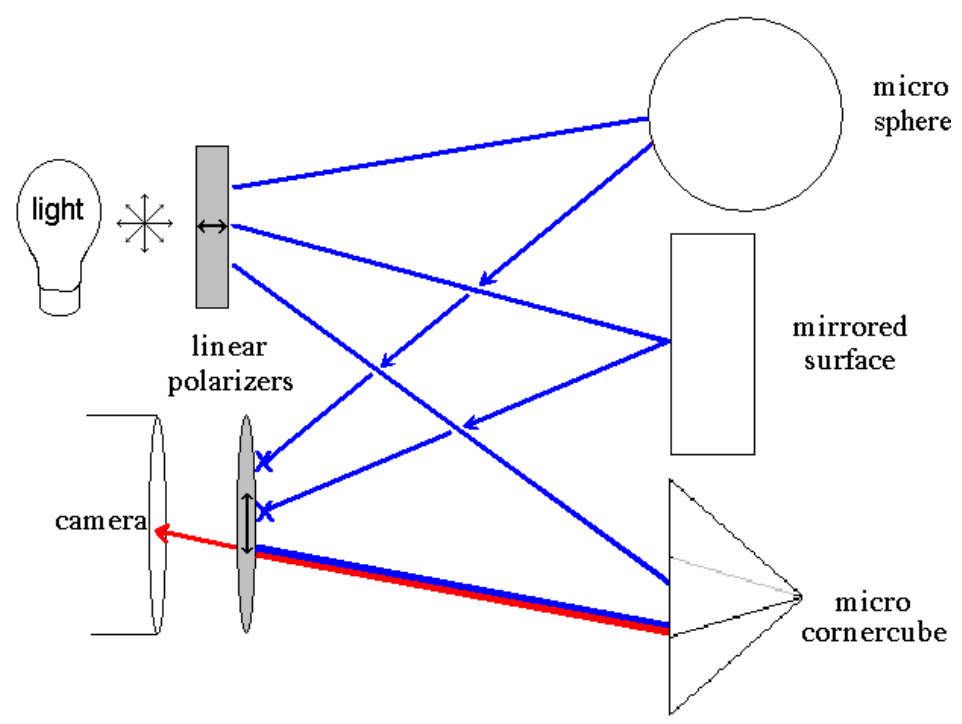

Figure 1. Reflected polarization return from three different types of surfaces. Linearly polarized incident light is shown in blue. Reflected light retaining the same polarization is shown in red. Light reflecting with altered polarization is shown in green. Spatial orientation of the linear polarizers is indicated by arrows.

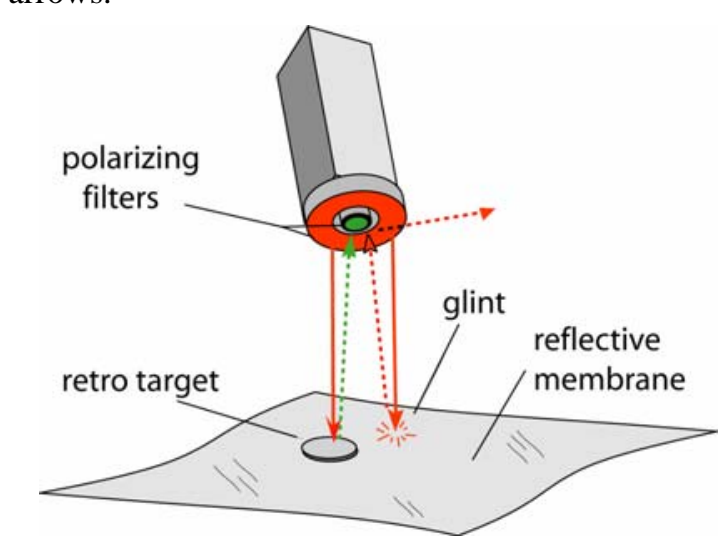

a)

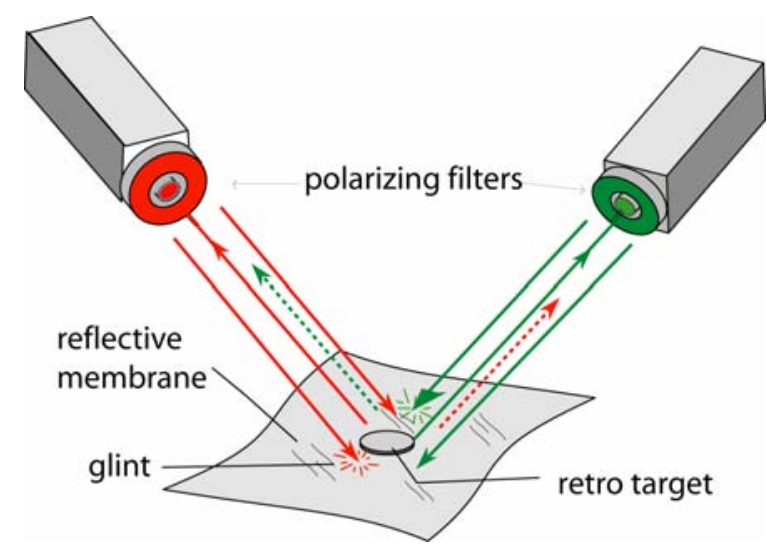

b)

Figure 2. Cross polarization signal discrimination by a) coaxial crossed polarization, and b) microsphere coaxial aligned polarization. For polarization discrimination, vertical polarization is shown in red, horizontal polarization in green. Figure b) also shows the setup for color selectivity. For the case of color selectivity alone, these colors reflect the actual colors of light used for each coaxial LED/camera set.

Two conceptually distinct signal enhancement techniques utilizing polarization were tested for reducing glint from images. Furthermore, a noise reduction method utilizing chromatic filters to select a different color bandpass for each camera was tested. The chromatic filters were then added to the coaxial aligned polarization setup, for a synergistic effect. Table 1 summarizes all the different configurations reported in the paper. The following paragraphs discuss each case in detail.

Figure 2a shows the coaxial crossed polarization technique: Cornercube retroreflectors receive linearly polarized light, but return randomly depolarized light. On the other hand, glints from the reflective membrane surface retain approximately the same polarization orientation as the illumination light. The camera is fitted with a second linear polarizer oriented perpendicular to the polarizer on the LED array. Thus, all light 
maintaining the original polarization orientation (including glints) is blocked from reaching the camera by the second polarizer. In this setup, numerous cameras and illumination angles do not interfere with one another, as long as all camera polarizers are parallel to one another, and perpendicular to all illumination source polarizers.

Figure 2b shows the coaxial aligned polarization technique: Each coaxial camera and LED array set are filtered by linear polarizers oriented in parallel to one another. Coaxial camera/LED sets viewing the test article at opposite angles also have polarizers oriented in parallel to each other, but perpendicular to the first camera/LED polarizer set. This scheme allows only light from the coaxial LED array to reach a camera. For most surface geometries, glints appear more easily from opposing illumination sources than from a coaxial source. Unless there is a surface normal to the camera axis, surface glints will not appear. This setup works best with a two-camera system, but may also be used in a 3-camera system, although with lessened noise reduction. For this arrangement, microsphere retroreflective targets were used.

Table 1. Filter and target configurations for each technique.

\begin{tabular}{|c|c|c|c|c|c|}
\hline Technique & $\begin{array}{c}\text { target } \\
\text { type }\end{array}$ & $\begin{array}{c}\text { R LED } \\
\text { filter }\end{array}$ & $\begin{array}{c}\text { R camera } \\
\text { filter }\end{array}$ & $\begin{array}{c}\text { L LED } \\
\text { filter }\end{array}$ & $\begin{array}{c}\text { L camera } \\
\text { filter }\end{array}$ \\
\hline coaxial crossed polarization & $\mathrm{CC}$ & $\mathrm{P}_{\mathrm{V}}$ & $\mathrm{P}_{\mathrm{H}}$ & $\mathrm{P}_{\mathrm{V}}$ & $\mathrm{P}_{\mathrm{H}}$ \\
coaxial aligned polarization & sphere & $\mathrm{P}_{\mathrm{V}}$ & $\mathrm{P}_{\mathrm{V}}$ & $\mathrm{P}_{\mathrm{H}}$ & $\mathrm{P}_{\mathrm{H}}$ \\
color selectivity & sphere & red & red & green & green \\
color + aligned polarization & sphere & red $+\mathrm{P}_{\mathrm{V}}$ & red $+\mathrm{P}_{\mathrm{V}}$ & green $+\mathrm{P}_{\mathrm{H}}$ & green $+\mathrm{P}_{\mathrm{H}}$ \\
control experiments & either & none & none & none & none \\
\hline
\end{tabular}

Figure $2 \mathrm{~b}$ also illustrates the two-color selectivity technique. In this method, chromatic filters are substituted for the polarization filters. The net result is that each camera detects only light reflected from its coaxial, same color (red or green) illumination source. This method was investigated by itself and in combination with the coaxial aligned polarization method described previously.

\section{Experimental Setup}

The test article investigated was a $1 \mathrm{~m}^{2}$ aluminized gossamer membrane of 1 mil thick CP2 ${ }^{\circledR}$ polyimide. Two cameras were used in each experiment. Cameras were located a distance of $2 \mathrm{~m}$ from the test article and were oriented parallel to the floor at $45^{\circ}$ angles to the membrane and perpendicular to one another. The images and angles reflect real-use situations, and were not chosen to exaggerate the relative image improvement. Images were taken with two Pulnix model TM-6710 video cameras with Fujinon-TV lenses (model 606284; 1:1.8/75 and model 166535; 1:1.4/12.5) attached. Illumination came from Lumiled Luxeon ${ }^{\circledR}$ Ring LED arrays with either 12 (red, $\lambda=625 \mathrm{~nm}$, model LXHLND92 ), or $12+6$ (green, $\lambda=530 \mathrm{~nm}$, model LXHL-NM96) LEDs each. Tech Spec ${ }^{\mathrm{TM}}$ cellulose acetate butyrate film linear polarizers were used for polarizing illumination light. Camera images were polarized by Prinz ${ }^{\mathrm{TM}} 58 \mathrm{~mm}$ glass linear polarizers. The green LED array output was chromatically filtered by custom made 6" diameter glass filters (transmission in the range of $\lambda=440-530 \mathrm{~nm}$ ) made by Rainbow Research Optics. The unfiltered red LED light showed better chromatic exclusion from the green filtered 
camera than unfiltered green light exclusion from the camera + red (wratten \#23A) filter combination. RR Capture (C) version 1.00 software was used to record video images. All images are digitally unenhanced in linear recording and output modes. Pixel intensities, pixel areas, and line profiles within images were calculated by IMAQ ${ }^{\mathrm{TM}}$ Vision Builder 6 software (National Instruments). Values were summed up over 35 target points, and noise values included any regions outside the target areas with an intensity level over $2 \%$ of the target signal intensity, to account for random camera noise in the absence of any image detection.

Retroreflective targets were affixed in a 5 x 7 grid pattern on a $1 \mathrm{~m}$ x $1 \mathrm{~m}$ aluminized gossamer CP2 membrane produced by SRS Technologies. The 1/2 inch round targets were cut from $3 \mathrm{M}^{\mathrm{TM}} 7610$ microsphere retroreflective tape, and $3 \mathrm{M}^{\mathrm{TM}} 3000 \mathrm{X}$ microcorner cube retroreflective tape.

\section{Results and Discussion}

Signal and noise values were calculated by integrating the pixel intensities over the areas of targets (signal) or glint regions on the gossamer sail (noise). The coaxial aligned and coaxial crossed polarization, color selectivity, and color selectivity in conjunction with coaxial aligned polarization techniques described in the previous sections were each compared for noise reduction against unenhanced measurements. Figure 3 shows signalto-noise ratios for each technique. Microsphere retroreflectors were chosen as targets for the coaxial aligned polarization technique, since their greater flexibility and lighter weight make them an obvious choice over cornercube retroreflectors when the specific depolarization property of the cornercubes becomes unimportant.

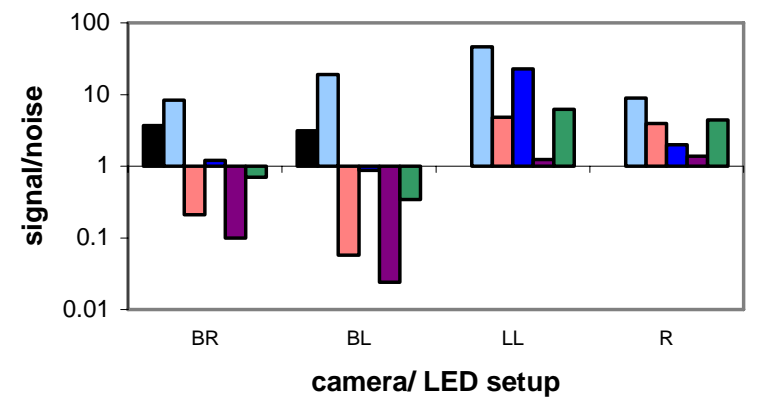

a)

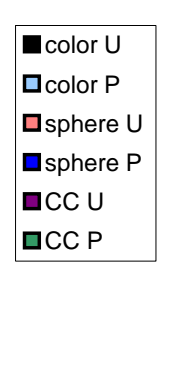

b)

Figure 3. Signal-noise ratios for three configurations a) raw data, and b) normalized for control (unfiltered) values. Camera/LED setups labeled in the $\mathrm{X}$ axes were $\mathrm{BR}=$ both cameras, LEDs on the right side only; BL=both cameras, LEDs on the left only; LL=left camera only, LEDs on left; RR=right camera, LEDs on right side. Bar colors represented the same categories for both figures a) and b). In the figure legend describing which noise reduction technique was used, "color" signifies color selectivity, "sphere" signifies microsphere retroreflectors with coaxial aligned polarization, "CC" signifies microcornercube retroreflector with coaxial cross polarization, "U" signifies unpolarized images, and "P" signifies polarized.

Placing a linear polarizer on a camera results in a drop in intensity, even when the camera polarizer is parallel to the incoming light polarization. This effect necessitated opening the camera aperture according to the brightness of the signal. In some cases, the 
target intensities differed between polarized and unpolarized images, even after adjusting for this effect. Another signal intensity inequality existed between right-left camera perspectives. Glints observed in the right camera from illumination on the left were greater than left-right observation. For this reason, normalization of the data was calculated by comparing the raw polarized data for each technique and camera/LED setup against unpolarized data of the same setup.

Another measure of the impact of glints is to measure the total area of the image that is saturated by a glint. Total glint areas per sail is given in Figure 4a, since the total measurable sail region lost by glint obscuring is just as important as signal-to-noise ratios for quantifying effective noise reduction. Figure $4 \mathrm{~b}$ shows the ratio of glint area to nonglint sail membrane area.

Measuring glints produced by illumination from the opposing side, coaxial illumination, and both together demonstrated that image glints arose mostly from opposing illumination sources. This is best demonstrated by the sphere U and CC U bars in the BR and BL setups in Figure 3a signal to noise data. The same result is shown by the LR and RL bars vs. the LL and RR bars in the glint area data in Figure 4a. The sail membrane was unavoidably subject to air currents within the room, which had a small, but detectable effect upon the localization of glints. Another small source of variation came from decreasing the camera f-stop when adding polarizers or chromatic filters to manually adjust for reduced signals.

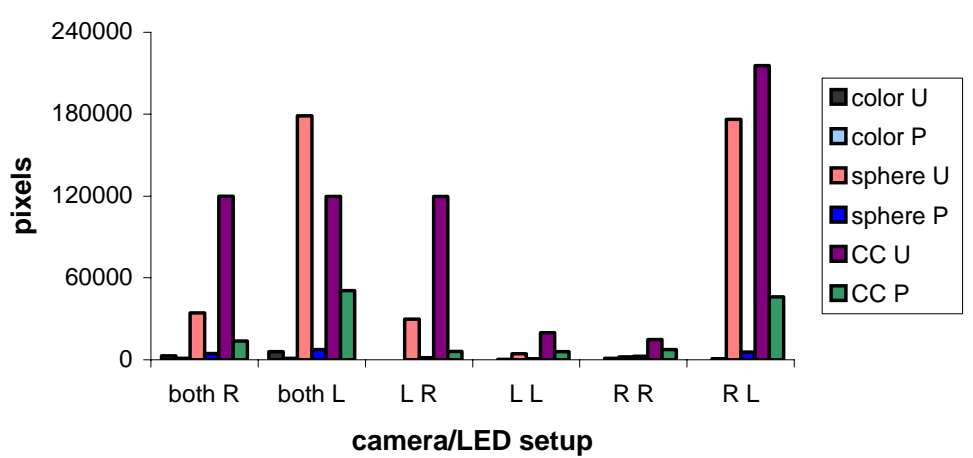

a)

Figure 4. Glint area a) raw data, and $\mathrm{b}$ ) ratio of glint area to dark, nontarget membrane surface area. $\mathrm{X}$ axes and figure legend definitions same as in Figure 3. The $\mathrm{Y}$ axis in Figure a) is in linear scale, compared to logarithmic scale in all other graphs. This emphasizes the magnitude of the enhancements achieved by the different methods. Figure b) data is not normalized for control values.

Color selectivity combined with polarization enhanced the signal-to-noise ratio as high as 800 fold in one setup, as compared to the same conditions without filters. Signalto-noise enhancements $(\mathrm{E})$ using both illumination sources was calculated by the formula $\mathrm{E}=\operatorname{antilog}\left[\Sigma^{\mathrm{k}} \log (\mathrm{S} / \mathrm{N}) / \mathrm{k}\right]$,

where $\mathrm{k}$ is the number of individual $\mathrm{S} / \mathrm{N}$ ratios averaged per calculation. These logarithmically averaged values were: color + polarization $=115$ times, color alone $=30.9$ times, coaxial aligned polarization $=9.3$ times, and coaxial crossed polarization $=$ 10.0 times. Average glint area reductions for each technique using both illumination sources (normalized for control values) are: color + polarization $=99.1$ times, color alone 
$=24.4$ times, coaxial aligned polarization $=18.0$ times, and coaxial crossed polarization $=$ 3.71 times.

\section{Color selectivity and polarization}

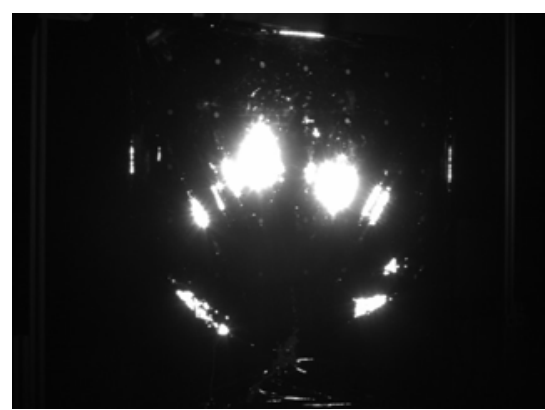

a)

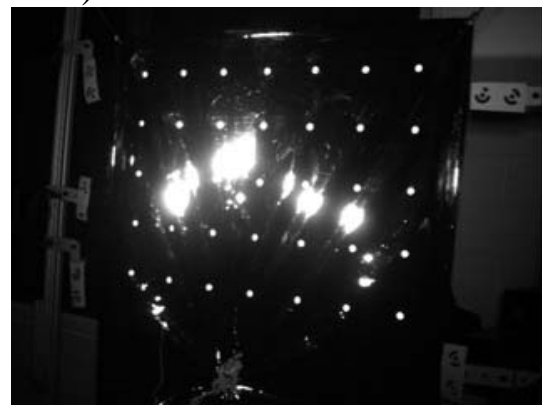

d)

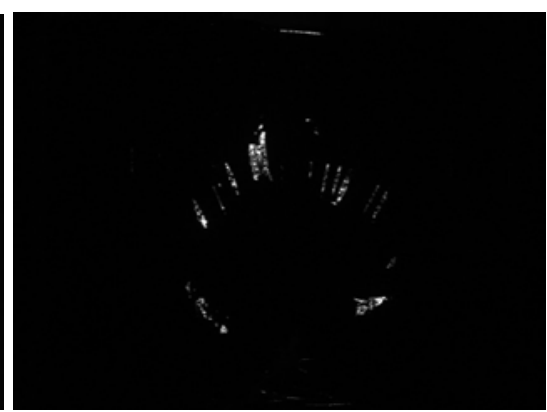

b)

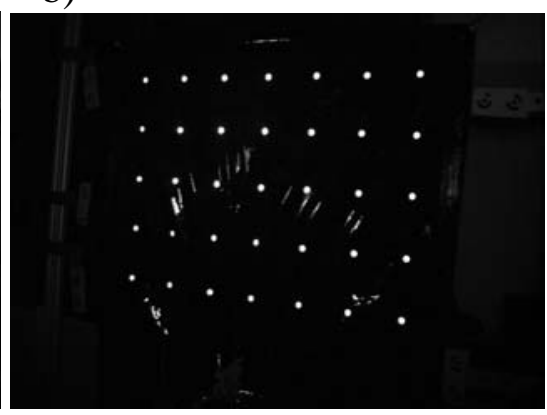

e)

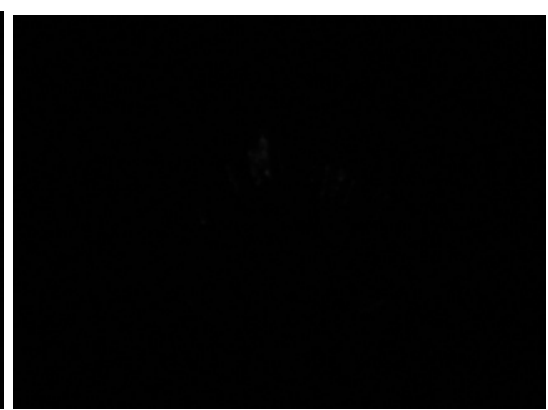

c)

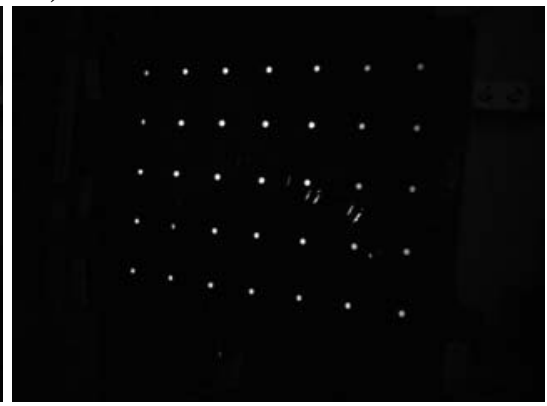

f)

Figure 5. Glints from unpolarized, polarized, and 2 color + polarization setups. Top row: illuminated from the opposing side, Bottom row: Illuminated from both sources a) and d) no noise reduction; b) and e) coaxial aligned polarization; c) and f) polarization + color selectivity

A severe case of opposing illumination producing glints on an aluminized gossamer membrane is shown in Figure 5a. The membrane area is covered with large ridges and small creases. Ridges figure prominently in the appearance of glints with coaxial aligned polarization, by providing a normal surface to the camera axis, even when the camera is well away from viewing normal to the overall membrane. The circular reflection is due to the ring geometry of the LED array. Figure 5b shows a raw image demonstrating the enhancement due to the coaxial polarization scheme. Initially the glints in 5a were strongly saturated, thus the potential enhancement of image quality is even greater than shown here. Both glint area and intensity are dramatically decreased. Targets do not appear in these top three images, since all illumination is far off axis from the camera, therefore target retroreflection returns the light back in the opposing direction. Figure 5c shows the complete elimination of glint from the image when the coaxial polarization and color discrimination schemes are used in conjunction. Color selectivity alone did not eliminate all glints from images.

With coaxial + opposing illumination, retroreflective targets appear in Figures 5 d-f. Figure $5 f$ shows all 35 targets with very little glint present. Figure 5e shows great reduction of glint area with coaxial aligned polarization. Figure $5 d$ shows a typical glint obscured image with many targets washed out and others confused with small glint spots. 
Choosing better camera angles may remove all remaining glints, when using these glint removal techniques. Increasing image contrast of these raw images also eliminates the little remaining glint from Figure $5 f$.

\section{Reflectivity properties of cornercube and microsphere retroreflectors}

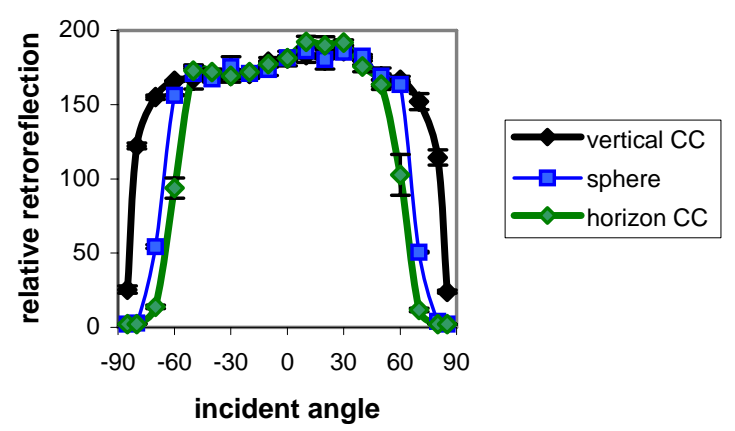

a)

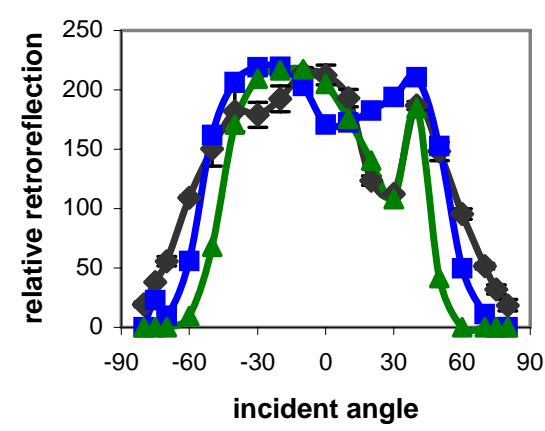

b)

Figure 6. Retroreflection return for cornercube and microsphere targets by angle. Relative average intensities of integrated target areas for targets at varying angles with respect to the camera. Figure a) is for a coaxial camera/LED array setup. Figure b) is for LED illumination at a $2^{\circ}$ offset from the camera - target axis.

Retroreflection efficiency depends upon the angle of incident light with respect to the normal of the target surface, and whether the alignment of the illumination and camera systems are coaxial, or offset by a small angle. Retroreflection dependence upon incident angle is shown in Figure 6 for a) coaxial, or b) offset $\left(2^{\circ}\right)$ geometries. The dip in the graph of Figure $6 \mathrm{~b}$ was caused by the camera and LED array axes being offset in a particular direction. The dip was more pronounced in cornercubes as has been previously noted ${ }^{1}$. The cornercube reflectance has a preferential orientation with respect to its cross hatching pattern. This is due to the geometry of the three facets of the cornercube that light reflects from. Thus, to obtain target uniformity in the images, coaxial illumination is very important to use in photogrammetry experiments using both microsphere retro or cornercube retro targets.

One consideration in choosing target material is that the cornercube tape produced by $3 \mathrm{M}^{\mathrm{TM}}$ has a visible cross hatching pattern on its face. This cross hatching gives a distinct pattern of light and dark regions in photogrammetric targets, as shown by the partially saturated line profile in Figure 7. This pattern lowers the quality of these retroreflectors as a targeting material, since it reduces centroiding accuracy of the photogrammetric software. Non-uniformity of light/dark cornercube target regions occurs, due to the line profile being intentionally taken off-axis of the cross hatching pattern. The intensity fluctuations of microsphere targets are small compared to the average pixel intensity, thus photogrammetric software centroiding is more accurate. On the other hand, patterned fluctuations of the target area intensity, such as seen with the cornercube tape, can be smoothed out by defocussing the camera. 


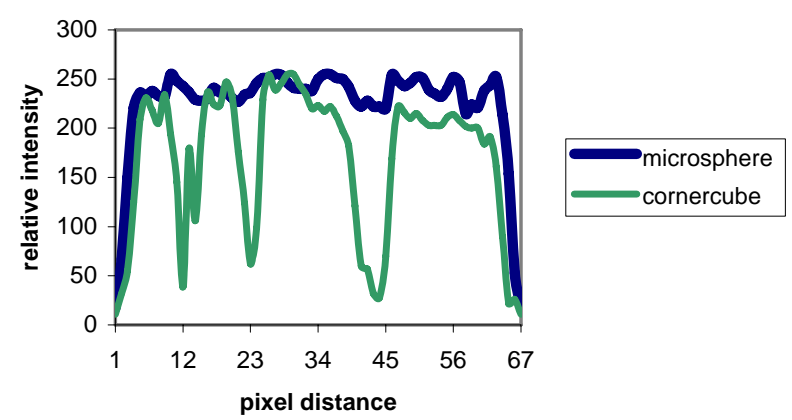

Figure 7. Typical line profile of cornercube and microsphere target intensity for 1 inch diameter targets. Maximum pixel intensities just below saturation level. Relative intensities of separate profiles are arbitrary and do not actually indicate that one technique gives brighter than another.

\section{Conclusion}

Chromatic filters and linear polarizers are simple, yet highly effective means of removing glints from images of reflective test articles. The coaxial aligned and coaxial crossed polarization techniques reduced image noise to similar degrees. Glints deriving from high surface curvature or depolarized light reflecting off rough metallic surfaces were the remaining sources of noise. Color selectivity alone eliminated glints slightly better than polarization techniques. The slight drawbacks of color selectivity, namely higher cost of filters and greater heating, would not prohibit its use for many applications. Color selectivity also does not lessen its efficiency of noise reduction in the presence of roughened metallic surfaces. Combining color selectivity and coaxial aligned polarization by far provided the highest glint rejection and highest signal-to-noise ratio. Photogrammetric measurements of reflective test articles, which were previously prohibited by glints, are clearly enabled by the use of polarization and color selectivity, especially in conjunction with each other. These noise reduction techniques may save considerable overall processing time, otherwise spent enhancing contrast of poor quality images.

\section{References}

${ }^{1}$ O'Brien D.C., Faulkner G.E., Edwards D.J., "Optical Properties of a Retroreflecting Sheet", Applied Optics Vol. 38, No. 19, 1999, pp. 4137-4144

${ }^{2}$ Scholl M.S., "Ray Trace Through a Corner-cube Retroreflector with Complex Reflection Coefficients", J. Opt. Soc. Am. A-Opt. Image Sci. \& Vision Vol. 12 No. 7, 1995, pp. 1589-1592

${ }^{3}$ Garwood B., "Sensing in the Retroreflective Mode", Banner Engineering Corp. [online tutorial], URL:http://63.85.138.133/literature_resources/tutorial/old/4b_retro_mode.html

${ }^{4}$ Siemens Energy \& Automation, Inc., "Photoelectric Controls: 1-4", [online tutorial] URL: www.sea.siemens.com/contrlbu/upld/files/p146-149.pdf 
${ }^{5}$ Morris J.W. and An C.H., "Properties of Non-symmetric Retroreflectors", in Polarimetry: Radar, Infrared, Visible, Ultraviolet and X-Ray, J.W. Morris and R.A. Chipman, eds., Proc. SPIE Vol. 1317, Oct. 1990, pp. 333-345

${ }^{6}$ Azzam R.M.A. and Liu J., "Polarization Properties of Cornercube Retroreflectors: Theory and Experiment", Appl. Opt. Vol. 36, No. 7, 1997, pp. 1553-1559 